\title{
Correlation between Chemical Glass Components and the Glass Sticking on Sputtered PtIr Physical Vapour Deposition Coatings for Precision Blank Moulding
}

\author{
Kirsten Bobzin, Nazlim Bagcivan, Tobias Brögelmann, Tobias Münstermann \\ RWTH Aachen University, Surface Engineering Institute, Aachen, Germany \\ Email: info@iot.rwth-aachen.de
}

Received 2 February 2014; revised 6 March 2014; accepted 21 March 2014

Copyright (C) 2014 by authors and Scientific Research Publishing Inc.

This work is licensed under the Creative Commons Attribution International License (CC BY). http://creativecommons.org/licenses/by/4.0/

\section{Abstract}

The increasing demand on high quality optical systems with complex geometries, low tolerances and a low installation space necessitates new replicative production systems for complex optical glass elements. The technology precision blank moulding shows promising properties to comply with these demands on an industrial bulk production. Due to the required high surface quality and low surface roughness of produced optical elements, moulding dies must have comparable low roughness and defect-free surfaces. To reduce wear and chemical interaction with the hot glass, moulding dies are often coated with a thin sputtered physical vapour deposition (PVD) coating. The objective of this research work was to analyze the diffusion behaviour inside different industrially used low-Tg (transformation point) glasses and their interaction with three different noble metal coating systems during an application oriented heating test. Therefore, three different PtIr coating systems with different interlayers $(50 \mathrm{~nm}$ nickel as reference, $20 \mathrm{~nm}$ chromium, without interlayer) were deposited and tested in combination with six different industrially used low-Tg glasses. Using energy-dispersive X-ray spectroscopy (EDS) a diffusion of the light alkali and alkaline earth metals (sodium, potassium, calcium) was detected. The interaction between glass and coating was analyzed by EDS, scanning electron microscopy (SEM) and X-ray diffraction (XRD). The different chemical compositions of the glasses have a significant influence on the interaction between glass and coating system. Several correlations between the chemical composition of the glasses and the amount of glass adhesion on the three coating systems were identified. The percentage of ions allocated to network modifiers lithium oxide, sodium oxide and potassium oxide correlates with the intensity of the interaction between coating and glass. The intensity of glass adhesion on the reference coating system PtIr/Ni is related with the zinc content in the glasses. Due to a diffusion process of the nickel interlayer, a direct correlation between the zinc content in the glasses and glass adhesion exists. The coating system with chromium interlayer showed com- 
parable results to the system without interlayer.

Keywords

PtIr, PVD, Glass Adhesion, Precision Blank Moulding

\section{Introduction}

In the last decades optical industry has become a growth market. The increasing demand for high-quality complex optical components requires new production technologies. Conventional production methods as grinding and polishing are not suitable for the bulk production of complex high quality optics. The new technology, precision blank moulding, introduced in the 70's in Japan [1]-[3] shows potential to fulfil the high demands concerning surface quality, low form tolerances and cost effectiveness. During an isothermal moulding process, a glass gob is formed between two ultra-precise moulding dies to a finished optical component without any postprocessing. The aim of this production technology is to generate a complex optical component with low form tolerances and a high surface quality in a single step. The limiting factor for the productivity of the process is the degradation of the tool surface due to thermal and mechanical loads and chemical interactions between the tool and the hot glass during the moulding process. To protect the cemented carbide moulding dies from glass adhesion, wear and oxidation a PVD (physical vapour deposition) or PECVD (plasma enhanced chemical vapour deposition), coating is used [4]-[6]. Different ceramic coating systems like TiAlN, CrN, CrWN [7]-[9] as well as diamond like coatings (DLC), for example Me-C:H with a metal-rich sublayer (Ti, Cr, Si, ‥) [10] and noble metal coatings on the basis of platinum and iridium (Pt-Ir, Pt-Re, Ir-Re) [3] [11]-[15] are used. The most widespread and industrially used coating is a noble metal system based on platinum and iridium deposited by MSIP (magnetron sputter ion plating) technology [5] [16]. A metallic interlayer is used below the noble metal coating to prevent diffusion of the binder element $(\mathrm{Co}, \cdots)$ from the substrate to the coating surface and to increase the compound adhesion between substrate and coating [3]. $\mathrm{Cr}, \mathrm{Ni}$ and $\mathrm{Ti}$ interlayer are used in combination with the noble metal coatings. A comparison between the different interlayer systems was carried out regarding the adhesion strength given in literature. The substitution of the Ni-interlayer by a Cr-interlayer contributes to increasing compound properties significantly [16] [17]. The development of a PtIr/Cr coating system and a correlation between the interlayer thickness and the compound adhesion have been described in previous work [13] [17]. Research work regarding the system properties of noble metal coating systems, which set chemical composition of industrially used low Tg-glasses in correlation with interactions between glass and coatings was done for a pure iridium coating [18], but is still missing for Pt-Ir coating systems. The objective of this research work was to compare three noble metal Pt-Ir coating systems with different interlayers ( $\mathrm{Ni} / \mathrm{Cr} /-)$ concerning their interactions with six industrially used low-Tg glasses of different chemical composition. The industrially used coating system PtIr/Ni represents the reference system with an interlayer thickness of $50 \mathrm{~nm}$. The coating PtIr/Cr with an interlayer thickness of $20 \mathrm{~nm}$ was developed in previous works and was proven sufficient for an improved compound adhesion [13] [17] in comparison to the reference specimen. Additionally, a system without interlayer was considered in this study. Chemical interactions between the coating and the glass were analyzed by EDS measurements of the glass and the coating surface after heating tests in contact with the glasses. The diffusion of elements inside the glass was analyzed by EDS measurements on polished cross sections of the annealed glasses. In this way, the elements with high mobility were identified. These results were correlated with XRD measurements of the coating inside and outside the glass contact zone. To determine the formation of additional phases inside the coating system as function of the measurement depth, different incidence angles were used. The peaks were analyzed according DeKeijser and Langford [19]-[21] method to determine the crystallite size and lattice strain parameters.

\section{Experimental Details}

\subsection{Coating Deposition}

A coating unit LH Z400, Leybold Heraeus GmbH, Cologne, Germany was used for deposition of the three coat- 
ing systems PtIr/Ni, PtIr/Cr and PtIr. A pure nickel target (99.99\%) and a pure chromium target (99.7\%) with a diameter of $75 \mathrm{~mm}$ were used for the deposition of the interlayer and a $\mathrm{Pt}_{40} \mathrm{Ir}_{60}$-target with the same diameter for deposition of the functional layer. The distance between surface of the specimens and target was kept constant at $50 \mathrm{~mm}$. Polished, binderless cemented carbide CTM00A, Ceratizit, Empfingen, Germany was used as substrate. It consists of tungsten carbide with a medium grain size $<2.5 \mu \mathrm{m}$ and a Co-binder content $<0.4 \%$ [22]. Preparation of the specimens includes a cleaning process in a six bath ultrasonic washing unit followed by a rinse cycle with isopropanol and a drying process with filtered compressed air. Inside the coating chamber an additional plasma etching process was used to activate and prepare the surface for coating deposition and to ensure sufficient compound adhesion. Deposition parameters are shown in Table 1. To evaluate thickness and morphology SEM-micrographs of fractured cross sections were taken (ZEISS DSM 982 Gemini, Carl Zeiss Microscopy GmbH, Jena, Germany).

\subsection{System Properties: Application Oriented Heating Test}

The diffusion behaviour inside the glass specimen and the interaction between the glass and coating surface was analysed by an application-oriented annealing test. Analysis were carried out using six commercial glasses N-FK5 and P-LASF47, Schott AG, Mainz, Germany, L-BAL42 and L-LAM60, Ohara Inc., Oyama, Japan, K-VC89 and K-PSFn1 produced by Sumita Optical Glass Inc., Saitama, Japan. All glasses are low-Tg glasses designed for glass moulding applications. The annealing temperatures were set to ensure a comparable viscosity of $\eta=8.5 \mathrm{dPa} \cdot \mathrm{s}$ for each glass. The holding temperatures were $\mathrm{T}_{\mathrm{H} 1}=590^{\circ} \mathrm{C}(\mathrm{L}-\mathrm{BAL} 42), \mathrm{T}_{\mathrm{H} 2}=615^{\circ} \mathrm{C}$ (P-LASF47, L-LAM60, K-VC89, K-PSFn1) and $\mathrm{T}_{\mathrm{H} 3}=645^{\circ} \mathrm{C}(\mathrm{N}-\mathrm{FK} 5)$. The holding time was kept for $4 \mathrm{~h}$ with a heating rate of $10^{\circ} \mathrm{C} / \mathrm{min}$. The annealing was done in a low-pressure nitrogen atmosphere with a chamber pressure between 0.1 and 1 Pa using a high vacuum furnace PVA MOV 553T, PVA TePla, Wettenberg, Germany. The chemical composition and the diffusion behaviour of the glasses were determined by EDS measurements of polished cross sectional samples of the glasses before and after annealing tests using a detector by Bruker Nano $\mathrm{GmbH}$, Berlin, Germany. Additional information about the chemical composition of the glasses produced by Schott AG was taken from data sheets. Crystallographic phases of the annealed coating specimens were analysed by XRD using a XRD 3003 by General Electrics, Munich, Germany equipped with a Cu-X-ray tube $\left(\lambda \mathrm{CuK} \alpha=1.540598 \times 10^{-10} \mathrm{~m}\right)$. The grazing incidence method (GID) was used to detect the phase composition of thin coatings. A variation of the incidence angle gave information about the phase composition depending on the position along the cross section. On the basis of XRD measurements with an incidence angle $\omega=2^{\circ}$ a peak analysis according to DeKeijser and Langford [19]-[21] was done to determine the domain size D and the lattice strain $\varepsilon$ inside the coating systems in as deposited state and after annealing tests inside and outside the glass contact zone. Therefore, the bragg-peaks $\langle 111\rangle$ and $\langle 200\rangle$ of the PtIr mixed crystal related to a diffraction angle $39.8^{\circ}<2 \theta_{\langle 111\rangle}<40.7^{\circ}$ and $46.2^{\circ}<2 \theta_{\langle 200\rangle}<47.3^{\circ}$ were fit by a pseudo-Voigt function. The integral peak width $\beta$ of the pseudo-Voigt profile is described by Equation (1) [20].

$$
\frac{2 \omega}{\beta}=\left(\frac{\sqrt{\pi}}{2 \sqrt{\ln 2}}+\eta\left(\frac{\pi}{2}-\frac{\sqrt{\pi}}{2 \sqrt{\ln 2}}\right)\right)^{-1}
$$

$2 \omega$ describes the full width at half maximum (FWHM) of the fitted peak and $\eta$ the weighting coefficient of the pseudo-Voigt function. The integral breadths of the Lorentzian and Gaussian components can be obtained using Equations (2) and (3) [19].

Table 1. Deposition parameters of the three coating systems deposited on the laboratory coating unit LH Z400.

\begin{tabular}{ccccc}
\hline Parameter & Unit & Etching & Deposition Interlayer Ni/Cr/- & Deposition PtIr \\
\hline Temperature & {$\left[{ }^{\circ} \mathrm{C}\right]$} & 350 & 350 & 350 \\
Basepressure & {$[\mathrm{Pa}]$} & $3 \times 10^{-3}$ & $3 \times 10^{-3}$ & $3 \times 10^{-3}$ \\
Pressure & {$[\mathrm{Pa}]$} & - & 0.5 & 0.5 \\
Cathode power & {$[\mathrm{W}]$} & -1024 & $-41 /-24 /-$ & 50 \\
Bias voltage & {$[\mathrm{V}]$} & & & 41 \\
\hline
\end{tabular}




$$
\begin{gathered}
\frac{\beta_{L}}{\beta}=a_{0}+a_{1} \eta+a_{2} \eta^{2} \\
\frac{\beta_{G}}{\beta}=b_{0}+b_{1 / 2} \sqrt{1+b \eta}+b_{1} \eta+b_{2} \eta^{2}
\end{gathered}
$$

where $a_{0}=0.017475, a_{1}=1.50484, a_{2}=-0.534156, b_{0}=0.18446, b_{1 / 2}=0.812692, b_{1}=-0.659603$, $a_{2}=-0.534156, b_{0}=0.18446, b_{1 / 2}=0.812692, b_{1}=-0.659603, b_{2}=0.445542$ and $b_{1}=-0.998497$. The instrumental peak broadening of the Gaussian and Lorentzian sub-function were determined using a lanthanum hexaboride standard powder with a grain size of $10 \mu \mathrm{m}$ as reference material. The structural integral peak breadth $\beta_{L}^{f}$ and $\beta_{G}^{f}$ for the Lorentzian and the Gaussian sub-function of the structural profile $f$ are described by Equations (4) and (5) including the integral breadth of the fitted profile $h$ and the reference profile g [19].

$$
\begin{gathered}
\beta_{L}^{f}=\beta_{L}^{h}-\beta_{L}^{g} \\
\left(\beta_{G}^{f}\right)^{2}=\left(\beta_{G}^{h}\right)^{2}-\left(\beta_{G}^{g}\right)^{2}
\end{gathered}
$$

According to the Scherrer equation (Equation (6)) the domain size $D$ is related to the integral breath $\beta_{L}^{f}$ of the size broadened profile. Regarding Equation (7) the lattice strain $\varepsilon$ is obtained by the integral breath $\beta_{G}^{f}$ of the strain broadened profile.

$$
\begin{gathered}
D=\frac{\lambda}{\beta_{L}^{f} \cos \theta} \\
\varepsilon=\frac{1}{4} \beta_{G}^{f} \cot \theta
\end{gathered}
$$

\section{Results}

\subsection{Coating Properties}

Figure 1 shows SEM micrographs of the three deposited coating systems. All three coatings show a columnar morphology. Due to the similar morphology and the thin layer thickness the nickel and chromium interlayer are only visible as a thin line between substrate and toplayer.

The determined coating thicknesses are listed in Table 2. Due to identical coating parameters of the top layer (see Table 1), the thickness of the interlayer can be determined by the subtraction of the thickness of PtIr coating from the total thickness of PtIr/Ni and PtIr/Cr.

\subsection{Chemical Composition and Diffusion Behaviour of Tested Glasses}

The glass N-FK5 has the smallest amount of different chemical components and is the only glass, which contains fluorides and oxides. It includes only potassium as cations of network modifier and no intermediates. The two glass P-LASF47 and K-VC89 are characterised by a high content of zinc cations which can act as network former or modifier. The networks of both glasses are based on lanthanum oxide and silicon dioxide. K-PSFn1 is the only silicon free glass, which is based on a phosphorus pentoxide and germanium dioxide network. The glasses L-BAL42 and L-LAM60 by Ohara Inc. contain at least three network modifiers and several intermediates. The chemical components of the tested glasses are listed in Table 3.

SEM micrographs of the polished cross sections of the Schott glasses are shown in Figure 2. The corresponding results of the EDS measurements are listed in Table 4. A reduction of the element potassium is detected near the glass surface of N-FK5 (points 1-1 and 1-2). The reduction decreases with increasing distance to the glass surface (points 1-3 and 1-4). Measurements in the cross section of the glass P-LASF47 reveal no significant change of the chemical composition. At the outer area some glass bubbles were generated during the annealing test.

EDS measurements of both glasses produced by Ohara Inc. reveal a reduction of the sodium content in the glass near to the surface after the annealing test. The chemical composition of the bulk material remains unchanged. The glass L-LAM60 shows a local phase separation at the outer glass area. In the light phase, visible in 
Table 2. Coating thickness of the three deposited coating systems PtIr/Ni, PtIr/Cr and PtIr.

\begin{tabular}{cccc}
\hline & PtIr/Ni (Ref) & PtIr/Cr & PtIr \\
\hline Interlayer & Nickel & Chromium & - \\
Total coating thickness[nm] & 330 & 300 & 280 \\
Interlayer thickness $[\mathrm{nm}]$ & 50 & 20 & - \\
\hline
\end{tabular}



Figure 1. SEM micrographs of the three coating systems PtIr/ Ni (a), PtIr/Cr (b) and PtIr (c).
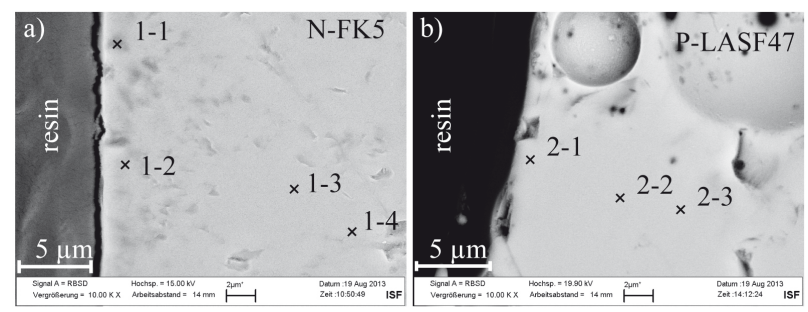

Figure 2. Polished cross sectional specimen of the glasses N-FK5 (a) and P-LASF47 (b) produced by Schott AG after annealing tests.

the SEM micrograph, silicon and zinc content are reduced and lanthanum and gadolinium content are increased (point 4-1). The polished cross sectional specimens of both glasses are shown in Figure 3. The results of the EDS measurements are listed in Table 5.

The polished cross sectional specimens of the glasses produced by Sumita Inc. are visible in Figure 4. The corresponding EDS measurements are listed in Table 6. The glass K-VC89 produced by Ohara Inc. shows only a reduction of the sodium content near the glass surface. The glass K-PSFn1 reveals a macroscopical discoloration into white, which is explained by a separation of the complete glass into two different phases visible in the SEM-micrograph in Figure 4(b).

The bright phase (point 6-5) has a higher density due to higher niobium content. The dark phase (point 6-4) has a lower density due to high phosphorus content. Three area measurements were done to determine glass composition at different positions independent of the described phase separation. The results indicate a reduction 
Table 3. Chemical components of the six industrial glasses divided into the cations of network former, network modifier, intermediates and the containing anions under specification of the cations proportion.

\begin{tabular}{|c|c|c|c|c|c|c|c|}
\hline & & N-FK5 & P-LASF47 & L-BAL42 & L-LAM60 & K-VC89 & K-PSFn1 \\
\hline \multirow{2}{*}{ Anions } & $\mathrm{O}$ & • & • & • & - & • & • \\
\hline & $\mathrm{F}$ & - & & & & & \\
\hline \multirow{6}{*}{$\begin{array}{c}\text { Cations of the } \\
\text { network former } \\
\text { [at.-\%] }\end{array}$} & $\mathrm{Si}$ & 69.6 & 15.1 & 63.3 & 23.0 & 1.9 & \\
\hline & $\mathrm{La}$ & & 22.6 & & 20.0 & 21.9 & \\
\hline & $\mathrm{Sb}$ & $<1.0$ & $<1.0$ & & & & \\
\hline & $\mathrm{P}$ & & & & & & 31.2 \\
\hline & $\mathrm{Ge}$ & & & & & & 4.9 \\
\hline & B & $* *$ & $* *$ & $*$ & $*$ & $*$ & $*$ \\
\hline \multirow{7}{*}{$\begin{array}{l}\text { Cations of the } \\
\text { network modifier } \\
\text { [at.-\%] }\end{array}$} & $\mathrm{Li}$ & & $* *$ & $*$ & $*$ & * & * \\
\hline & $\mathrm{Na}$ & & & 5.4 & 5.8 & 11.5 & 9.0 \\
\hline & $\mathrm{K}$ & 30.4 & & 1.1 & & & 8.1 \\
\hline & $\mathrm{Ca}$ & & & & 7.2 & & \\
\hline & $\mathrm{Sr}$ & & & & & & 3.1 \\
\hline & $\mathrm{Ba}$ & & & 17.7 & 4.7 & & \\
\hline & $\mathrm{Nb}$ & & 6.9 & & & 5.3 & 29.7 \\
\hline \multirow{8}{*}{$\begin{array}{c}\text { Cations of the } \\
\text { intermediates } \\
\text { [at.-\%] }\end{array}$} & $\mathrm{Zn}$ & & 32.2 & 5.5 & 10.7 & 35.1 & \\
\hline & $\mathrm{Ta}$ & & 10.1 & & 2.7 & 5.0 & \\
\hline & $\mathrm{Zr}$ & & 4.4 & & 6.2 & 6.9 & \\
\hline & $\mathrm{Y}$ & & 4.3 & & 8.1 & & \\
\hline & $\mathrm{Al}$ & & 4.2 & 7.0 & & & \\
\hline & Gd & & & & 11.5 & 7.8 & \\
\hline & $\mathrm{W}$ & & & & & 4.6 & 6.6 \\
\hline & $\mathrm{Bi}$ & & & & & & 7.5 \\
\hline
\end{tabular}

*: Not detectable; **: included, but not detectable; $\bullet$ : containing anions.

Table 4. EDS measurements on the polished cross sectional specimens of the glasses N-FK5 and P-LASF47 (Figure 2) after annealing tests.

\begin{tabular}{|c|c|c|c|c|c|c|c|c|c|}
\hline \multirow{2}{*}{ Point } & \multicolumn{9}{|c|}{ Cations of network former, modifier and intermediates [at.-\%] } \\
\hline & $\mathrm{Si}$ & $\mathrm{La}$ & $\mathrm{K}$ & $\mathrm{Zn}$ & $\mathrm{Nb}$ & Та & $\mathrm{Zr}$ & $\mathrm{Y}$ & $\mathrm{Al}$ \\
\hline $1-1$ & 82.7 & & 17.3 & & & & & & \\
\hline $1-2$ & 81.8 & & 18.2 & & & & & & \\
\hline $1-3$ & 77.1 & & 22.9 & & & & & & \\
\hline $1-4$ & 75.6 & & 24.4 & & & & & & \\
\hline $2-1$ & 14.8 & 22.7 & & 32.9 & 6.5 & 10.6 & 4.2 & 4.2 & 4.0 \\
\hline $2-2$ & 14.6 & 22.3 & & 32.2 & 7.4 & 9.8 & 4.6 & 4.6 & 4.6 \\
\hline $2-3$ & 15.7 & 23.0 & & 31.1 & 7.0 & 9.9 & 5.0 & 4.4 & 4.5 \\
\hline
\end{tabular}




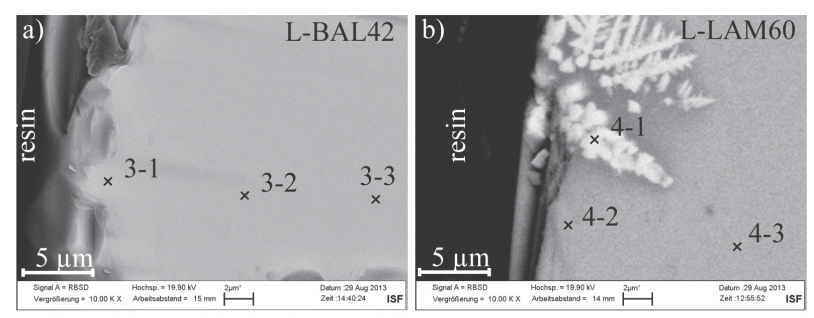

Figure 3. SEM micrograph of the polished cross sectional specimens of the glasses L-BAL42 (a) and L-LAM60 (b) produced by Ohara Inc. after annealing tests.

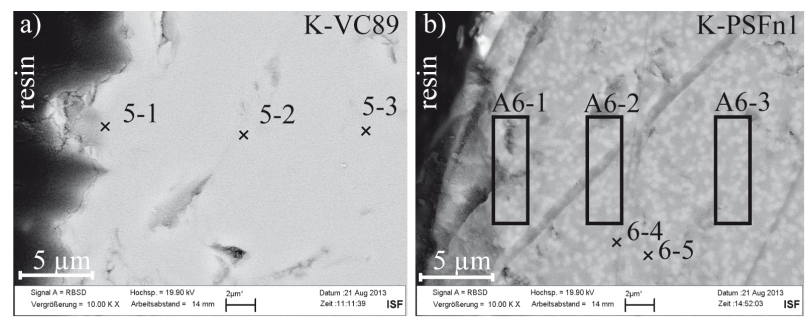

Figure 4. SEM micrograph of the polished cross sectional specimen of the glasses K-VC89 (a) and K-PSFn1 (b) produced by Sumita Inc. after annealing tests.

Table 5. EDS measurements on the polished cross sectional specimens of the glasses L-BAL42 and L-LAM60 (Figure 3) after the annealing test.

\begin{tabular}{ccccccccccccc}
\hline Point & \multicolumn{10}{c}{ Cations of network former, modifier and intermediates [at.-\%] } \\
& $\mathrm{Si}$ & $\mathrm{La}$ & $\mathrm{Na}$ & $\mathrm{K}$ & $\mathrm{Ca}$ & $\mathrm{Ba}$ & $\mathrm{Zn}$ & $\mathrm{Ta}$ & $\mathrm{Zr}$ & $\mathrm{Y}$ & $\mathrm{Al}$ & $\mathrm{Gd}$ \\
\hline $3-1$ & 64.2 & & 3.7 & 0.9 & & 19.0 & 4.8 & & & & 7.3 \\
$3-2$ & 64.5 & & 4.6 & 1.1 & & 17.5 & 5.1 & & & & 7.3 \\
$3-3$ & 62.3 & & 5.4 & 1.0 & & 19.4 & 5.2 & & & & 6.8 \\
$4-1$ & 4.9 & 48.0 & 3.3 & & 3.8 & 2.1 & 1.7 & 0.4 & 1.8 & 10.8 & & 23.2 \\
$4-2$ & 26.0 & 18.8 & 3.1 & & 8.0 & 5.0 & 11.3 & 3.0 & 6.1 & 7.8 & & 11.0 \\
$4-3$ & 25.8 & 20.4 & 5.2 & & 7.4 & 4.8 & 8.5 & 1.6 & 6.5 & 8.5 & & 11.1 \\
\hline
\end{tabular}

Table 6. EDS measurements on the polished cross sectional specimens of the glasses K-VC89 and K-PSFn1 (Figure 4) after the annealing test.

\begin{tabular}{|c|c|c|c|c|c|c|c|c|c|c|c|c|c|c|}
\hline \multirow{2}{*}{ Point } & \multicolumn{14}{|c|}{ Cations of network former, modifier and intermediates [at.-\%] } \\
\hline & $\mathrm{Si}$ & $\mathrm{La}$ & $\mathrm{P}$ & Ge & $\mathrm{Na}$ & $\mathrm{K}$ & $\mathrm{Sr}$ & $\mathrm{Nb}$ & $\mathrm{Zn}$ & $\mathrm{Ta}$ & $\mathrm{Zr}$ & Gd & $\mathrm{W}$ & $\mathrm{Bi}$ \\
\hline $5-1$ & 2.4 & 22.8 & & & 7.0 & & & 3.2 & 36.5 & 5.9 & 8.2 & 8.3 & 5.7 & \\
\hline $5-2$ & 2.4 & 23.0 & & & 7.7 & & & 3.9 & 35.7 & 5.0 & 9.3 & 8.0 & 5.1 & \\
\hline $5-3$ & 0.9 & 22.6 & & & 10.2 & & & 3.7 & 35.6 & 5.0 & 8.4 & 8.1 & 5.4 & \\
\hline A6-1 & & & 31.0 & 4.8 & 7.4 & 8.6 & 3.3 & 31.2 & & & & & 6.9 & 6.7 \\
\hline A6-2 & & & 31.1 & 5.0 & 7.2 & 8.2 & 3.3 & 30.1 & & & & & 6.6 & 7.1 \\
\hline A6-3 & & & 30.8 & 4.8 & 7.2 & 8.8 & 3.3 & 29.9 & & & & & 7.6 & 7.7 \\
\hline $6-4$ & & & 34.5 & 5.9 & 9.7 & 6.8 & 2.6 & 25.1 & & & & & 5.8 & 9.3 \\
\hline 6-5 & & & 29.2 & 4.1 & 8.0 & 9.0 & 3.0 & 32.7 & & & & & 7.3 & 6.5 \\
\hline
\end{tabular}


of sodium content in all three measurement areas (areas A6-1, A6-2, A6-3) independent of the position.

\subsection{Interactions between Glass and Coating}

The micrographs of the three coating surfaces, which were in contact with the six glasses during the annealing tests are shown in Figure 5. Due to the similar functional PtIr layers, the chemical composition of the six different industrially used glasses and the different interlayers of the PtIr coatings systems play the key role concerning interactions between glass and coating system. A comparison of the three micrographs of each glass reveals significant, interpretable differences. In general, the coating system PtIr/Ni induces the most residues with the six tested glasses. The coating systems PtIr and PtIr/Cr perform similar concerning glass adhesion and discoloration. The glass K-PSFn1 causes a similar amount of remaining glass on all three coating systems. The glass N-FK5 generates comparable glass adhesion on PtIr/Ni and PtIr/Cr.

In Figure 6 SEM-micrographs of the surface of the PtIr/Cr and a PtIr/Ni coating system in contact with the glass L-BAL42 after heating tests are visible. The low amount of glass adhesion generated with L-BAL42 enables a good comparability between the coatings' analysis of interlayer diffusion. The topography of the cemented carbide is still visible on both specimens due to the low coating thickness. In contrast to PtIr/Cr (Figure 6(a)), the grain boundaries of the substrate are clearly detectable as dark lines on the micrograph of PtIr/Ni (b). Existence of $\mathrm{Pt}_{40} \mathrm{Ir}_{60}$ functional top layer can be verified by EDS. Additionally enrichment of nickel and oxygen in the areas marked with the arrows were detected on PtIr/Ni. These Ni-rich phases are visible on all specimens of the coating system PtIr/Ni after heating tests. A comparable diffusion behaviour is not detectable on any specimens with the coatings PtIr/Cr or PtIr.



Figure 5. Micrographs of coating surfaces of PtIr/Ni, PtIr/Cr and PtIr after the application-oriented heating tests in contact with six industrially used low-Tg glasses. 


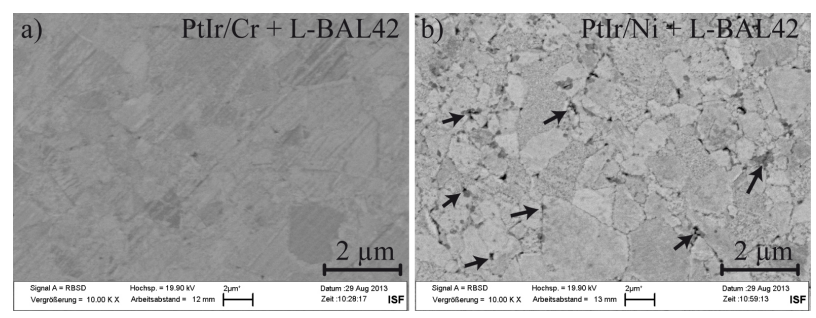

Figure 6. SEM-micrograph of the surface of the PtIr/Cr (a) and $\mathrm{PtIr} / \mathrm{Ni}$ (b) coating system after the heating test in the glass contact zone.

EDS measurements of all coating surfaces reveal no diffusion of the cations out of the glass into the coating. All measurements show comparable results for the measurements inside the contact area and outside the area of glass contact.

Figure 7, Figure 8 and Figure 9 illustrate the results of the XRD measurements of the three coating systems after heating tests. The specimens tested with N-FK5 are shown as example due to their comparable low amount of glass adhesion. The results on the left side are measured inside the contact area to the glass, the right side outside to the contact area. The 3D plot shows the x-ray diffractogram depending on the incidence angle $\omega$, which correlates with the measurement depth. The incidence angle was varied between $\omega=0.5^{\circ}$ and $\omega=3.5^{\circ}$. All measurement series contain WC peaks coming from the substrate at high incidence angles. Peaks at low incidence angles have a very low intensity with a high peak broadening due to the measuring technique. With higher $\omega$ the intensity increases by decreasing peak width. Both measurement series of the coating system PtIr/Ni (Figure 7) reveal two characteristic peaks of the mixed PtIr crystal between $2 \theta=39.8^{\circ}$ and $40.7^{\circ}$ for the $\langle 111\rangle$ orientation and between $2 \theta=46.2^{\circ}$ and $47.3^{\circ}$ for the $\langle 200\rangle$ orientation. Near the substrate the $\langle 111\rangle$ peak is superposed by a smaller peak of the intermetallic phase $\mathrm{Ni}_{2} \mathrm{~W}_{4} \mathrm{C}$ at $2 \theta=41.64^{\circ}$, visible in Figure 7 (b) outside the contact area at $\omega=3^{\circ}$. In the surface near area of the measurements inside the glass contact area an additional peak at $2 \theta=37.0^{\circ}$ is visible at inclination angle between $\omega=1.0^{\circ}$ and $2.5^{\circ}$, visible in Figure 7(a) inside the glass contact area at $\omega=2.0^{\circ}$. Parallel to this a small peak is detectable at $43.0^{\circ}$. Both peaks can be attributed to $\mathrm{NiO}$, a reaction product of oxygen from the glass and nickel from the interlayer.

The XRD measurement series of the coating system PtIr without interlayer are shown in Figure 8. Both diffractogramms inside (a) and outside the contact area (b) contain no additional intermetallic phases due to a reaction between glass or atmosphere and coating.

Figure 9 illustrates the XRD measurement series of the coating system PtIr/Cr inside and outside the glass contact zone after annealing tests. The measurements show significant differences concerning the phase composition. Both measurements have the same peaks resulting from mixed PtIr. The coating inside the glass contact area forms no additional phases besides PtIr and the WC at high incidence angles $\omega$. The diffractograms of the coating next to the glass contact zone show additional peaks at $2 \theta=37.5^{\circ}$ and $2 \theta=43.6^{\circ}$ which correlate with the cubic CrN phase. A formation of a chromium oxide phase $\left(\mathrm{Cr}_{2} \mathrm{O}_{3}\right)$ at $2 \theta=36.2^{\circ}$ is not detectable by XRD measurements.

Figure 10 illustrates the results of the peak analysis according to DeKeijser and Langford. The diagram shows the average of both crystal orientations. The black bars represent the domain size or lattice strain of the coating systems before the annealing test. All coating systems have a similar domain size and strain. After annealing tests larger grains are detectable for all systems. The reference coating system PtIr/Ni shows the most intensive grain growth up to a domain size of $45 \mathrm{~nm}$. The grain growth for PtIr and PtIr/Cr are similar with lower values. The lattice strain of the coating systems PtIr/Cr and PtIr decreases during the annealing. PtIr/Ni generates an increasing strain inside the crystal structure during annealing. The domain size and strain inside and outside the glass contact area are of a similar size, except for the strain of the coating system PtIr.

\section{Discussion}

The annealing experiments reveal a correlation between the diffusion behaviour of the glasses and the containing elements. As expected, network modifiers show the highest mobility of the three types of glass components. Especially the network modifiers of the first and second main group have a high mobility due to the single (al- 

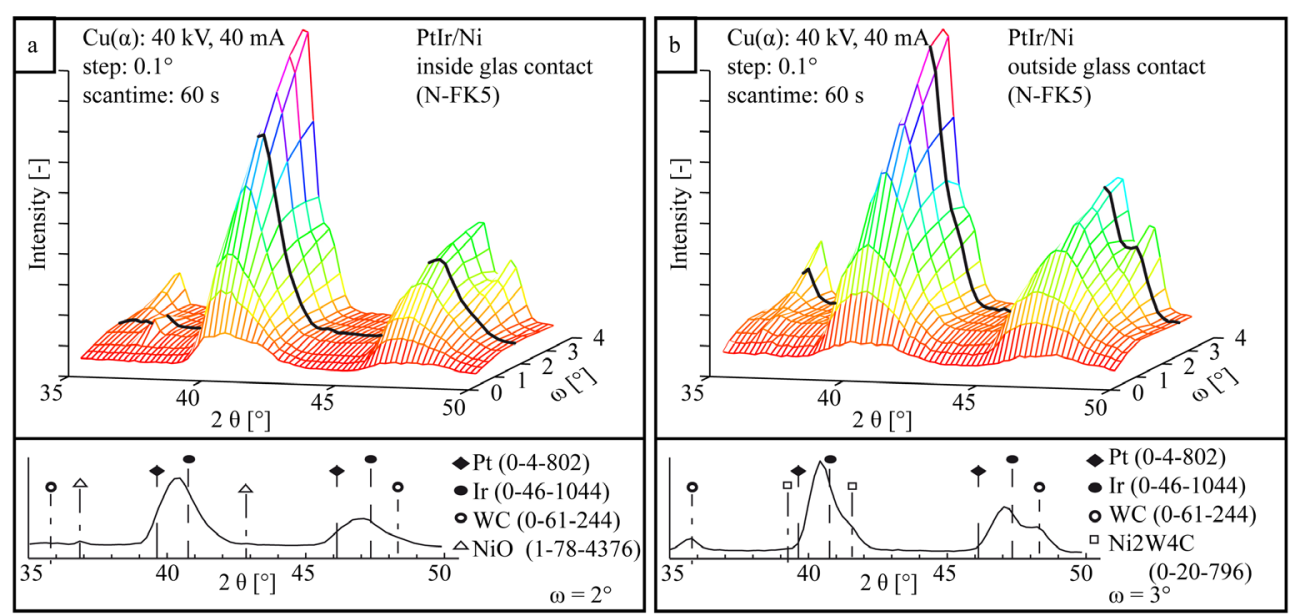

Figure 7. XRD diffractograms in dependency of the incidence angle $\omega$ of the reference coating system $\mathrm{PtIr} / \mathrm{Ni}$ inside (a) and outside (b) the glass contact area.
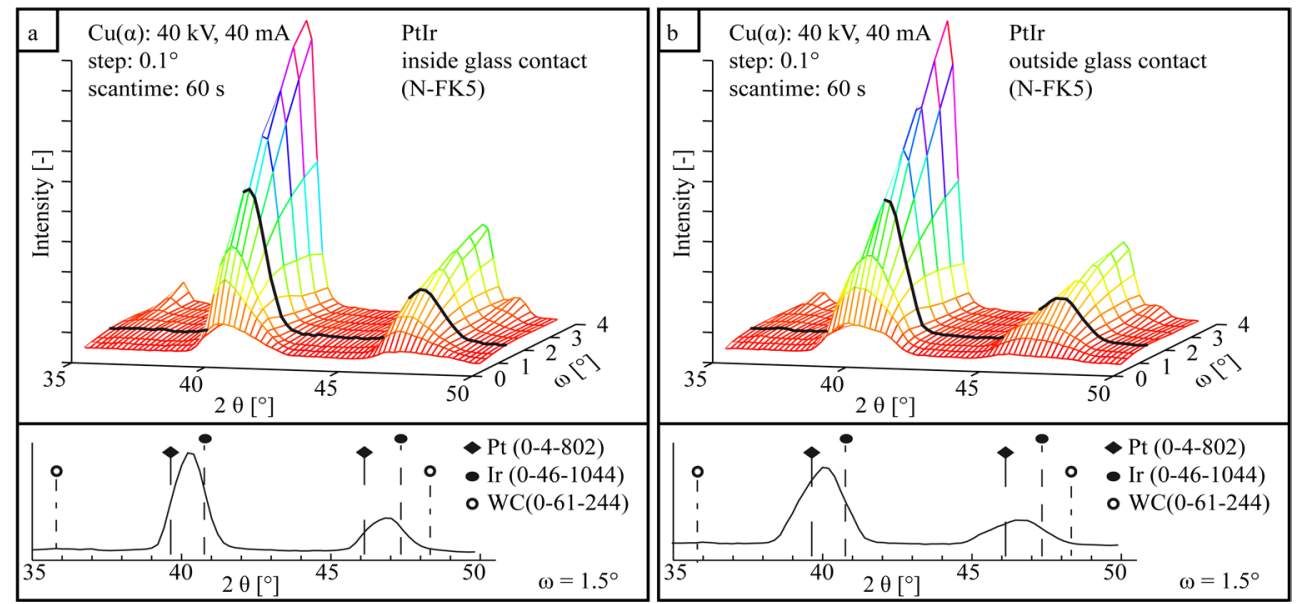

Figure 8. XRD diffractograms in dependency of the incidence angle $\omega$ of the coating system PtIr inside (a) and outside (b) the glass contact area.

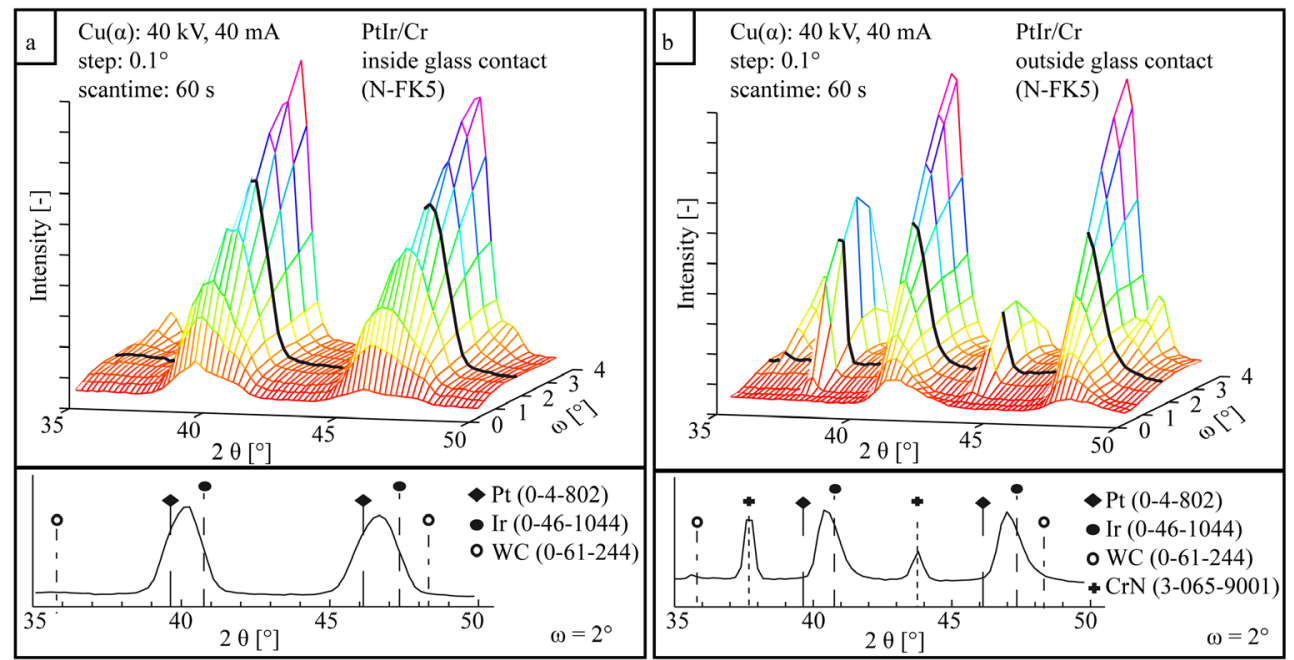

Figure 9. XRD diffractograms in dependency of the incidence angle $\omega$ of the coating system PtIr/Cr inside (a) and outside (b) the glass contact area. 


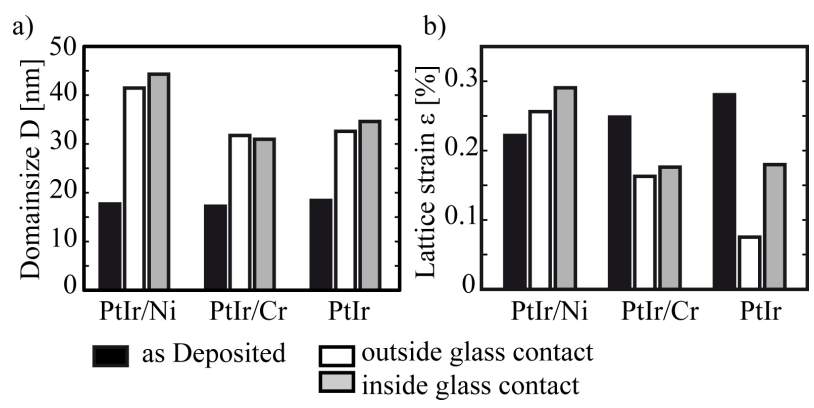

Figure 10. Domain size D (a) and lattice strain $\varepsilon$ (b) of the three coating systems PtIr/Ni, PtIr/Cr for the state as deposited, inside and outside the glass contact zone after the annealing test.

kali metals: Li, Na, K) and twice (alkaline earth metal: $\mathrm{Ca}, \mathrm{Sr}$ ) negatively charge. With increasing electronegativity and increasing ion diameter the mobility decreases. This explains the detected diffusion behaviour of the light alkaline elements sodium and potassium. According to this, it can be expected that lithium has an even higher susceptibility of diffusion in comparison to sodium, potassium, calcium, strontium or barium. This thesis is confirmed by the generation of bubbles inside the glass P-LASF47, which contains only lithium oxide as network modifier belonging to the critical group of alkaline or earth alkaline oxides (Table 3). Besides ions of the alkaline and alkaline earth metals, elements with a low evaporation temperature may be critical due to the higher mobility and reactivity. Figure 11 shows an extract of the vapour pressure data of selected elements, published by Honig [23]. For the used annealing parameters (pressure $\mathrm{p}_{\mathrm{H}}$ and holding Temperature $\mathrm{T}_{\mathrm{H}}$ ) the pure elements potassium, sodium, zinc are already in the vapour state. Due to this, it can be expected, that deoxidised zinc has a high probability to react with the coating surface.

Several correlations between the chemical composition and the glass adhesion are observable. A relationship between the network modifier of the alkaline and alkaline earth metal and the remaining glass on PtIr and $\mathrm{PtIr} / \mathrm{Cr}$ is visible. L-BAL42 shows the lowest amount of glass adhesion for all the tested coating systems. This glass contains only a small amount of sodium (5.4 at.-\%) and potassium (1.1 at.-\%). The third network modifier is barium. Barium is the heaviest, non radioactive alkaline earth metal. Due to its size and the twice negatively charge the mobility in the glass is quite low. In the glass L-LAM60 a slightly increased amount of the cations sodium in comparison to L-BAL42 is detectable. The glass sticking on the coatings PtIr and PtIr/Cr is also quite low. Only the combination with the coating system PtIr/Ni generates a distinct interaction and glass adhesion. For the glass P-LASF47 no alkaline or alkaline earth metal network modifier is detected by EDS, but on the basis of the datasheets it is known that the glass contains lithium. This might explain the existence of the glass adhesions on PtIr and PtIr/Cr. The glass K-VC89 has the highest amount of sodium (11.5 at.-\%), N-FK5 contains the highest amount of potassium (30.4 at.-\%) and K-PSFn1 contains both sodium (9 at.-\%) and potassium (8.1 at.-\%). All three glasses generate a significant amount of glass adhesion. The glass adhesions on the coating system PtIr/Ni can be set in correlation with the zinc content of the glasses. Both zinc-free glasses N-FK5 and K-PSFn1 show comparable results with all three coating systems. The zinc content in the glasses correlates with the amount of glass on the coating system with the nickel interlayer. L-BAL42 contains the lowest zinc content (5.5 at.-\%) and almost no glass adhesion is detectable. The glasses P-LASF47 and K-VC89 have the highest zinc content (32.2 at.-\%, 35.1 at.-\%) and in correlation to this the glass adhesion covers almost the complete contact area. The contact area of P-LASF47 is shaped as a ring due to the generation of a single gas pore. The generated amount of glass adhesion of L-LAM60 on PtIr/Ni is ranked between the glasses L-BAL42 and K-VC89 or P-LASF47 on the same coating system. This correlates with the zinc content of 10.1 at.-\%. A possible explanation for this correlation is a diffusion process of nickel out of the interlayer to the coating surface. In the SEM micrograph in Figure 6 the nickel rich phases can be detected along the grain boundaries of the substrate. The boundaries between the single tungsten carbides initiate irregularities in the coating system which act as diffusion path for nickel. In contact with oxygen of the glass the nickel oxidizes in the surface near area, which is detected in the XRD measurement in Figure 7(a) at incidence angles between $\omega=1.0^{\circ}$ and $\omega=2.5^{\circ}$. Due to the inert low pressure nitrogen atmosphere this oxidation is only detected in the contact area with the glass. At the 


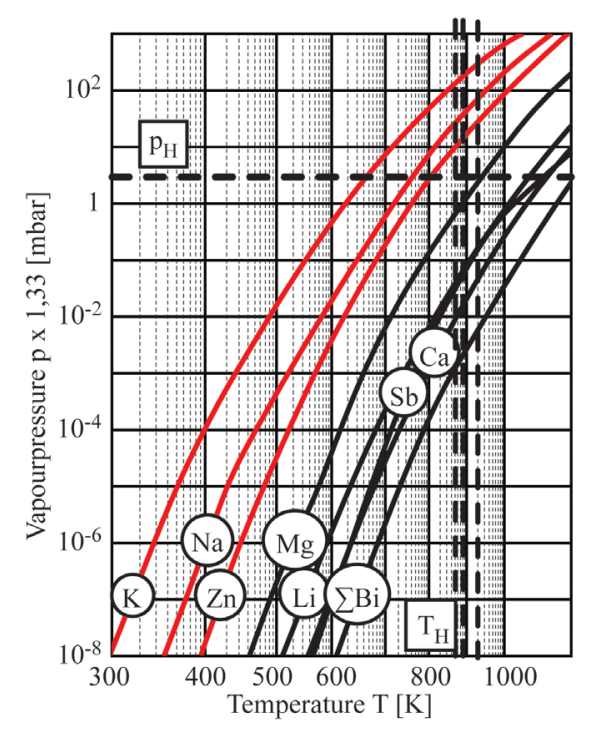

Figure 11. Vapour pressure data of selected elements [23].

interface between substrate and coating system the $\mathrm{Ni}_{2} \mathrm{~W}_{4} \mathrm{C}$ phase is detectable. The generation of a $\mathrm{Ni}_{2} \mathrm{~W}_{4} \mathrm{C}$ phase is described in literature during the sintering of WC with nickel binder [24] [25]. This phase may have a negative influence on the compound adhesion between substrate and coating. This thesis was not analysed in this research.

The increased strain inside the crystal structure, reported in Figure 10(b) confirms the diffusion of nickel across the PtIr coating. Additional nickel in the PtIr top layer introduces tension, which appears as strain of the crystal structure. A comparable diffusion of the element chromium to the coating surface is not detected. Neither the SEM micrograph in Figure 6, nor the XRD measurements in Figure 9(a) show diffusion or a generation of an oxide phase in contact with the glass. Probable reasons for this are the different diffusion pairs and the thinner interlayer thickness of PtIr/Cr. Due to the low pressure nitrogen atmosphere in the annealing experiments, chromium nitride is formed on the coating outside the glass contact area. With these results it cannot be said if the chromium diffuses to the coating surface or the nitrogen into the coating to the interlayer. The contact with the glass during the annealing process prevents a formation of this nitride phase in the glass contact zone. The diffusion behaviour of chromium generates no additional strain inside the top layer. Due to the annealing process the lattice strain is reduced as it can be seen in Figure 10(b). Additional to the lattice strain a low grain growth is important to keep the high quality of the functional surface. It is known that platinum tends to an intensive grain growth [26] [27]. An oversized grain growth will lead to a reduced surface quality. As it can be seen in Figure 10(a), the use of a thinner chromium interlayer reduces the grain growth of the PtIr top layer.

On the basis of the glass adhesion on the coating system PtIr/Ni in contact with the zinc containing glasses, it is expected, that zinc/zinc oxide reacted with nickel/nickel oxide. A reduction product of nickel and zinc could not be detected so far.

\section{Conclusions}

The interaction between six industrial low-Tg glasses and the three coating systems PtIr/Ni, PtIr and PtIr/Cr was analyzed after application oriented heating tests in a low pressure nitrogen atmosphere. To identify glass components with a high mobility, the chemical composition and the diffusion behaviour inside the glasses were analyzed. A correlation between the amount of the light cations of the alkaline metals and the glass adhesion on the coating systems PtIr and PtIr/Cr was identified. The amount of zinc content in the glasses has a direct influence on the glass sticking on the system PtIr/Ni. This glass adhesion can be attributed to a diffusion process of the interlayer nickel to the surface and chemical reactions between the nickel/nickel oxide phase and the zinc/zinc oxide phase. The coating system PtIr and PtIr/Cr did not show a comparable glass adhesion due to the zinc phase. Furthermore, a lower grain grows in comparison to the reference system PtIr/Ni and PtIr was detected for the system PtIr/Cr. 
A more detailed chemical analysis of the glass residue on the coating surface might help to understand interactions between glass and coating. To determine the influence of the interlayers on tool lifetime for precision glass moulding, application-oriented moulding experiments using industrially used glass presses will be carried out.

\section{Acknowledgements}

The author gratefully acknowledge the financial support of the Federal Ministry of Economics and Technology (BMWi) within the project "Coat4Glass-Wissensbasierte Schichtoptimierung für das Präzisionsblankpressen von Glasoptiken” at RWTH Aachen University.

\section{References}

[1] Angle, M.A., Blair, G.E. and Maier, C.C. (1974) Method for Molding Glass Lenses. US Patent No. 3833347.

[2] Hiramoto, T. (1980) Press Molding Method for Thick Optical Glass Molding. JP Patent 55062815.

[3] Monji, K.H., Aoki, M.M., Torii, H.H. and Okinaka, T.H. (1988) Mold for Press-Molding Glass Elements. US Patent No. 4721518.

[4] Brinksmeier, E., Glaebe, R. and Osmer, J. (2011) Surface Integrity Demands of High Precision Optical Molds and Realization by a New Process Chain. Procedia Engineering, 19, 40-43. http://dx.doi.org/10.1016/j.proeng.2011.11.077

[5] Ma, K.J., Chien, H.H., Chuan, W.H., Chao, C.L. and Hwang, K.C. (2008) Design of Protective Coatings for Glass Lens Molding. Key Engineering Materials, 364-366, 655-661. http://dx.doi.org/10.4028/www.scientific.net/KEM.364-366.655

[6] Torii, H., Aoki, M., Okinaka, H., Yuhaku, S. and Nakamura, S. (1986) Mold for Direct Press Molding of Optical Glass Element. US Patent No. 4606750.

[7] Kleer, G. and Doell, W. (1997) Ceramic Multilayer Coatings for Glass Moulding Applications. Surface and Coatings Technology, 94-95, 647-651. http://dx.doi.org/10.1016/S0257-8972(97)00518-5

[8] Hock, M., Schaffer, E., Doll, W. and Kleer, G. (2003) Composite Coating Materials for the Moulding of Diffractive and Refractive Optical Components of Inorganic Glasses. Surface and Coatings Technology, 163, 689-694. http://dx.doi.org/10.1016/S0257-8972(02)00658-8

[9] Lin, C., Duh, J. and Yau, B. (2006) Processing of Chromium Tungsten Nitride Hard Coatings for Glass Molding. Surface and Coatings Technology, 201, 1316-1322. http://dx.doi.org/10.1016/j.surfcoat.2006.01.064

[10] Brand, J., Gadow, R. and Killinger, A. (2004) Application of Diamond-Like Carbon Coatings on Steel Tools in the Production of Precision Glass Components. Surface and Coatings Technology, 180-181, 213-217. http://dx.doi.org/10.1016/j.surfcoat.2003.10.138

[11] Hagen, J., Burmeister, F., Fromm, A., Manns, P. and Kleer, G. (2009) Iridium Coatings with Titanium Sub-Layer Deposited by RF Magnetron Sputtering: Mechanical Properties and Contact Behavior with RoHS-Compliant Glass Melt. Plasma Processes and Polymers, 6, S678-S683. http://dx.doi.org/10.1002/ppap.200931701

[12] Kim, S.S., Kim, H.U., Kim, H.J. and Kim, J.H. (2007) Re-Ir Coating Effect of Molding Core(WC) Surface for Aspheric Glass Lens-Art. No. 671708. Optomechatronic Micro/Nano Devices and Components III, 6717, 71708-71708. http://dx.doi.org/10.1117/12.754329

[13] Bobzin, K., Bagcivan, N., Ewering, M., Brugnara, R.H. and Münstermann, T. (2012) Influence of Interlayer Thickness of a Thin Noble Metal MSIP-PVD Coating on Compound and System Properties for Glass Lens Moulding. Production Engineering, 6, 311-318. http://dx.doi.org/10.1007/s11740-012-0385-7

[14] Klocke, F., Bergs, T., Georgiadis, K., Sarikaya, H. and Wang, F. (2008) Coating Systems for Precision Glass Molding Tools. Proceedings of the 7th international conference THE Coatings, Chalkidiki, Greece, 1-3 October 2008, 209-218. http://dx.doi.org/10.4028/www.scientific.net/KEM.438.57

[15] Wu, F., Chen, W., Duh, J., Tsai, Y. and Chen, Y. (2003) Ir-Based Multi-Component Coating on Tungsten Carbide by RF Magnetron Sputtering Process. Surface and Coatings Technology, 163-164, 227-232. http://dx.doi.org/10.1016/S0257-8972(02)00616-3

[16] Klocke, F., Bouzakis, K.D., Georgiadis, K., Gerardis, S., Skordaris, G. and Pappa, M. (2011) Adhesive Interlayers' Effect on the Entire Structure Strength of Glass Molding Tools' Pt-Ir Coatings by Nano-Tests Determined. Surface and Coatings Technology, 206, 1867-1872. http://dx.doi.org/10.1016/j.surfcoat.2011.07.060

[17] Bobzin, K., Klocke, F., Bagcivan, N., Ewering, M., Georgiadis, K. and Munstermann, T. (2010) Impact Behaviour of PtIr-based Coatings with Different Interlayers for Glass Lens Moulding. Coatings in Manufacturing Engineering, 438, 57-64. http://dx.doi.org/10.4028/www.scientific.net/KEM.438.57 
[18] Bobzin, K., Bagcivan, N., Brögelmann, T. and Münstermann, T. (2013) Development and Qualification of a MSIP PVD Iridium Coating for Precision Glass Moulding. Materialwissenschaft und Werkstofftechnik, 44, 673-678. http://dx.doi.org/10.1002/mawe.201300174

[19] De Keijser, T.H., Mittemeijer, E.J. and Rozendaal, H.C.F. (1983) The Determination of Crystallite-Size and LatticeStrain Parameters in Conjunction with the Profile-Refinement Method for the Determination of Crystal-Structures. Journal of Applied Crystallography, 16, 309-316. http://dx.doi.org/10.1107/S0021889883010493

[20] Langford, J.I. (1987) Some Applications of Pattern Fitting to Powder Diffraction Data. Progress in Crystal Growth and Characterization, 14, 185-211. http://dx.doi.org/10.1016/0146-3535(87)90018-9

[21] De Keijser, T.H., Langford, J.I., Mittemeijer, E.J. and Vogels, A.B.P. (1982) Use of the Voigt Function in a SingleLine Method for the Analysis of X-Ray-Diffraction Line Broadening. Journal of Applied Crystallography, 15, 308-314. http://dx.doi.org/10.1107/S0021889882012035

[22] Ceratizit Austria GmbH, Hard Material Matters. 385-EN/DE 06.07 7001140. http://www.ceratizit.com

[23] Honig, R.E. (1957) Vapor Pressure Data for the More Common Elements. RCA Review, 18, 195-204.

[24] Liang, C. and Lin, S.T. (1997) Sintering of Injection-Moulded WC-7 wt\% Ni in a Hydrogen Atmosphere. Journal of Materials Science, 32, 3207-3212. http://dx.doi.org/10.1023/A:1018611003610

[25] Upadhyaya, G.S. (1998) Cemented Tungsten Carbides: Production, Properties, and Testing. William Andrew Publishing/Noyes, Westwood.

[26] Lee, D.S., Park, D.Y., Woo, H.J., Kim, S.H., Ha, J. and Yoon, E. (2001) Preferred Orientation Controlled Giant Grain Growth of Platinum Thin Films on $\mathrm{SiO}_{2} / \mathrm{Si}$ Substrates. Japanese Journal of Applied Physics, 40, L1-L3. http://dx.doi.org/10.1143/JJAP.40.L1

[27] Fischer, B., Goy, K., Kock, W., Lupton, D.F., Manhardt, H., Merker, J., Schoelz, F., Zurowski, B. and Goy, K.H. (1998) Dispersionsverfestiger Platin-Werkstoff, Verfahren zu seiner Herstellung und seine Verwendung. German Patent No. DE19714365-A1. 